\title{
To Live Long, Eat Less Salt: Salt Intake Reduction Promotion and Hypertension Control in China
}

\author{
Mei $\mathrm{Li}^{*}$, Liying Zhang and Jingjing Shi
}

The University of Hong Kong-Shenzhen Hospital, Shenzhen, China

\begin{abstract}
During the past decade, the prevalence of hypertension in Chinese adult population has increased dramatically. It's well accepted that hypertension is the main risk factor for the development of cardiovascular diseases (CVD). In order to control hypertension and CVD in China, addressing the behaviour risk factors in Chinese population is a cost-effective way. Among the known behaviour risk factors, salt intake reduction is one of the most cost-effective ways to control hypertension and CVD. Ecological Model was used in this review to analyse the pieces of evidence based determinants and barriers of salt consumption in China, which includes public policy, community, institutional, interpersonal and intrapersonal determinants. Aiming to prevent and control hypertension in China, efforts are needed to overcome these barriers. Some of the barriers are less or not at all modifiable (such as policy and culture), but most of them are possible to modify by behaviours changes. Recommendations for reducing population's salt consumption also be given based on Ecological Model. However, salt intake reduction would be more effective with the combined efforts of multi levels rather than individually.
\end{abstract}

Keywords: Salt intake reduction; Health promotion; Hypertension control

\section{Introduction}

Along with the rapid economic development and living standard improvement in China during the past decades, Chinese population has longer life expectancy than before [1]. However, great changes in lifestyle and eating habits have also happened among the Chinese population at the same time [2]. For examples, people are more prone to lead a sedentary life and eat westernized food (such as hamburger and chips). As a result, diet-related non-communicable diseases (NCDs) such as obesity, diabetes, and hypertension are become more and more common nowadays in China [3], which is so-called epidemiological transition in developing countries [4]. For China, $42 \%$ of death is caused by diet-related NCDs compared with all other death causes in 1995, and this proportion will reach $52 \%$ by 2020 [3].

Hypertension is a global public health issue [5] and it's well accepted that hypertension is the main risk factor for the development of cardiovascular diseases (CVD) [6]. According to a cross-sectional study in China, the prevalence of hypertension in Chinese adult population increased from $19 \%$ in 2002 [7] to $27 \%$ in 2013 , which means one in four adults in China has hypertension, and this percentage was only 5\% in 1960 [8]. In additional, the prevalence of hypertension in urban population is comparatively higher than that of in rural population [7].

The behaviour risk factors for hypertension including high salt consumption, alcohol and tobacco use and physical inactivity, among them, salt intake reduction is one of the most cost-effective ways to control hypertension and CVD [5]. In order to control hypertension and CVD in China, addressing the behaviour risk factors in Chinese population is a cost-effective way. Population-based intervention studies have shown that once salt intake was decreased, a reduction in population BP will be shown along with the decreased salt consumption [9]. It's reported that population-wide intervention for reducing salt consumption is very cost-effective (only US $\$ 10.6$ per DALY saved) [10], and salt intake reduction and tobacco use control can avoid 13.8 million death at the cost of less than one US dollars per person per year in developing countries over a decade [11].

Above all, hypertension is a public health issue in China; however, hypertension is also an avoidable disease by addressing behaviour risk factors, such as salt intake reduction. In this essay, the determinants and evidence base of salt consumption in China will be analysed, and context-specific recommendations on salt intake reduction will be given.

\section{Salt Consumption in China}

According to the definition of the healthy diet by WHO (2015), it's recommended that total salt intake should be less than $5 \mathrm{~g}$ per day. However, based on the salt consumption survey in Chinese residents in 2002, total salt intake was $10.7 \mathrm{~g}$ per person per day which is more than double of the WHO recommendation; the daily salt consumption was higher in rural population than urban population $(11.1 \mathrm{~g}$ and $9.7 \mathrm{~g}$ respectively); less than $20 \%$ people consumed less than $6 \mathrm{~g}$ salt per day [12]; and $70 \%$ of salt consumption was from table salt and nearly $10 \%$ from soy sauce added when cooking at home [13]. In order to address this issue, it is essential to analyse the barriers to salt intake reduction and set up appropriate interventions in China.

\section{Determinants and Evidence Based on Salt Consumption in China}

The salt consumption in Chinese population depends on a number of determinants which are explained as below. The understanding of the determinants is important in setting effective salt intake reduction promotion interventions. Table 1 shows the Ecological Model [14] to analyse the determinants of salt consumption in China.

*Corresponding author: Mei Li, The University of Hong Kong-Shenzhen Hospital, No.1, Haiyuan 1st Road, Futian District, Shenzhen, China 518000, Tel: (+86) 0755 86913333; Fax: (+86) 0755-86913108; E-mail: lim5@hku-szh.org

Received June 15, 2016; Accepted June 22, 2016; Published June 29, 2016

Citation: Li M, Zhang L, Shi J (2016) To Live Long, Eat Less Salt: Salt Intake Reduction Promotion and Hypertension Control in China. Health Care: Current Reviews 4: 169. doi: 10.4172/2375-4273.1000169

Copyright: (c) $2016 \mathrm{Li} \mathrm{M}$, et al. This is an open-access article distributed under the terms of the Creative Commons Attribution License, which permits unrestricted use, distribution, and reproduction in any medium, provided the original author and source are credited. 


\begin{tabular}{|l|l|}
\hline Factors & Determinants \\
\hline Public policy & Global, national and local policies, legislation, taxes, salt consumption recommendations, national programmes \\
\hline Community & Hospital, school, media, web site, diet cultures, cultural food environment, surroundings, location \\
\hline Institutional & Restaurant, supermarket, salt industry, food industry, marketing, package of food \\
\hline Interpersonal & Family, friends, social networks and peers \\
\hline Intrapersonal & $\begin{array}{l}\text { Age, gender, education, occupation, living place, married status, health status, income, nutrition knowledge, misconceptions, taste preference, attitudes, } \\
\text { eating habits, experiences of food }\end{array}$ \\
\hline
\end{tabular}

Table 1: Determinants of salt consumption in China.

\section{Evidence Based of Salt Consumption in China}

\section{Public policy}

In 2004, World Health Assembly (WHA) adopted the "WHO Global Strategy on Diet, Physical Activity, and Health". In 2013, WHO adopted "Global Action Plan for the Prevention and Control of Non-communicable Diseases 2013-2020", requiring the Member States to reduce 30\% salt/ sodium intake in mean population by 2015 . And in the same year, WHO endorsed the sodium intake guideline for adults and children [5]:

WHO recommends a reduction in sodium intake to reduce blood pressure and risk of cardiovascular disease, stroke and coronary heart disease in adults (strong recommendation). WHO recommends a reduction to $<2 \mathrm{~g} /$ day sodium $(5 \mathrm{~g} /$ day salt) in adults (strong recommendation). WHO recommends a reduction in sodium intake to control2 blood pressure in children (strong recommendation)?

Under the requirement of WHO, the Chinese government set up salt reduction policies in 2005, which was following the recommendations of "WHO Global Strategy on Diet, Physical Activity, and Health" [15]. In 2008, the Chinese government introduced voluntary guidelines, regulating those food industries should label the salt contain per $100 \mathrm{~g}$ or per serving on the food package. However, this guideline is not compulsory, most of the food industries didn't follow the guides as labelling salt contain will increase the cost of packaging.

There were two major national salt reduction programmes in China. The 'Government-led National Initiative of Health Lifestyle for All' campaign was launched in 2007, and the relevant health promotion activities including salt intake reduction have covered $63 \%$ of Chinese districts [16]. In 2011, the 'China Rural Health Initiative Sodium Reduction Study' was done in order to identify a novel, scalable strategy for salt intake reduction and control hypertension in rural China [17]. This study is still carrying on and according to the feedback; stronger evidence is needed for extensive change in salt policy in China.

\section{Community}

China is the large country with a huge population, and diet cultures and eating habits are different in different areas, which can affect the salt amount added in food during cooking. For example, people in some parts of China like to eat vegetable pickles (such as Pao Cai and Zha Cai) [18], and these pickles are added a lot of salt during the production process. Moreover, Chinese cooking techniques also include using salt to preserve food, for example, salted fish is popular in the coastal area, and salted meat is a common food in Sichuan and Hunan Provinces. Another salted food including salted eggs, salted chicken, etc. all these salted food is high is salt [19]. Different from western cooking way, Chinese people prefer to cook at home and use soy sauce when cooking, $70 \%$ of salt consumption was from table salt and nearly $10 \%$ of daily salt consumption is from soy sauce added during cooking [13]. Additionally, an interesting paper found that people are more prone to cook at home if they live in the community with no or few restaurants [20], which makes them easier to control their salt consumption.
In 2008, in order to teach people how to calculate the amount of salt when cooking, many local governments handed out free saltrestriction-spoons in the communities. The spoon is designed to hold $2 \mathrm{~g}$ table salt, so people are educated not to use more than 3 spoons of salt per person per day when cooking. It is been proved that free salt-restriction-spoons in community-based salt intake reduction promotion have positive effects in reducing the salt assumption and lower blood pressure of the population [21].

Moreover, media's effect on salt intake should not be omitted. Television watching and computer use can be another barrier for salt intake reduction, as children would be more prone to consume advertised foods such as fast food and snakes [22], which makes it hard to control the salt intake from these foods.

\section{Institutional}

Along with the changes in eating habits, the Chinese population is consuming more and more processed food nowadays, but most of the food industries do not have the willingness to reduce salt contain in their products because of commercial interests and inadequate incentives from the government [15]. In order to make food more attractive to consumers, restaurants always put extra seasoning inside the food. It's been reported that those people frequently eat in restaurants are related to less healthy eating styles, for example, prefer foods high in salt or fat [23].

In 2011, salt industries are encouraged by the government to produce low sodium salt and promoted the use of low sodium salt in restaurants, cafeterias and at homes and in 2015, low sodium salt has accounted for $22 \%$ of the sauces, cooked food, and seasonings consumed at home [24].

\section{Interpersonal}

According to the review from the free salt-restriction-spoons campaign in China, peer groups are useful in increasing residents' nutrition knowledge on why and how to control their salt consumption [21]. It's well accepted that eating habits and food choices can affect peoples' salt consumption. A study found that $94 \%$ of spouses included in the study have similar eating habits, and $19 \%$ of people have similar food choices with their peers [25]. Children's food choices and eating habits are greatly influenced by their parents' eating style [26] and also influenced by their peers [27].

There is limited literature was done in studying interpersonal determinants of salt intake in China, but based on author's working experiences as a doctor in China, if people has family members (especially their parents) or friends who are suffering hypertension, diabetes or CVD, then the people will be more careful about the healthy diet, as they can get more information about the relationship between high salt intake and hypertension, and their family members and friends will also remind them to take less salt now and then.

\section{Intrapersonal}

In the community-based salt intake reduction campaign, some 
people who had the free salt-restriction-spoons did not use it when cooking or did not know how to use it correctly. It has been revealed that $62 \%$ of the people got the free spoons but only $45 \%$ of the population were actually using it [28]. Chen et al. [24] hold a survey to understand the reason why people were not willing to use the spoon, and they found that those people who came from higher socioeconomic classes, had higher education, gained more knowledge about healthy diet, had relatively healthy eating habits were more likely to use salt-restrictionspoons in a correct way, especially for those people who had present hypertension or other NCDs. Another study on the consumption and sources of dietary salt showed that dietary salt intake in males is significantly higher than that of females, and people aged 18-59 years old group have the highest dietary salt intake comparing with other age groups. Living place is another impact factor of salt consumption, as the cooking habits in a different place would affect the salt and soy sauce amount added in cuisine. It is been reported that rural and suburban population consume more dietary salt than urban population [28]. Married status can also affect people's salt intake, as single people are prone to eat processed food or eat outside rather than cooking at home, which makes it hard to calculate the salt consumption.

In addition, according to author's experience, there are some misconceptions about salt intake among the Chinese population, for example, some people think that they should intake more salt if they sweat in summer, some people believe that salt has no harm at all as is a kind of natural seasoning, and some young people think that only old people need to take care of their diet.

\section{Recommendation for Salt intake Reduction in China}

The last section has analysed the evidence-based determinants and barriers to salt consumption in China. In order to prevent and control hypertension in China, efforts are needed to overcome these barriers. Some of the barriers are less or not at all modifiable (such as policy and culture), but most of them are possible to modify by behaviours changes.

\section{Public policy}

Centrally implemented, population-based approaches to salt reduction has been proved to be the most cost-effective strategy for CVD prevention in developing countries [29,30]. Develop national guidelines or standards for salt contain in processed food and seasonings according to WHO documents. Moreover, a government should give incentives to the food industry, restaurants to use low sodium salt as lots of studies show that replacing sodium with potassium in salt can reduce blood pressure [31-33], and it is also shown to be effective in a study hold in rural Chinese population trial [34]. However, incentives should be given to salt industry to produce low sodium salt to make sure them to join in the salt intake reduction program.

Decrease taxes and price for low sodium salt or increase taxed for high salt contained food. Taxation according to salt contain in food is shown to be effective in Finland; Enforce the implementation of the existing salt intake reduction related laws, for example, require all food industries should label the salt contain per $100 \mathrm{~g}$ or per serving on a food package. Labelling is an effective strategy in reducing salt consumption in European Community; Regulate and marketing control of high salt contain food, which is done in some European countries and is proved to be effective [35]; However, there are not many studies done in developing countries.

\section{Community}

Community-, school- and hospital-based education on healthy diet and nutrition knowledge, aiming to increase the awareness in the general population. Community-based education is highly effective in high salt intake population [9]. For example in some areas in China, pickles and salted food are popular and soy sauce is a common kind of seasonings when cooking, so community campaigns on reducing intake of pickles, salted food and soy sauce are proposed to be effective in those areas. Free cooking class in the community might be held inside the community, teaching people cooking skills and how to choose healthy food. Additionally, community health workers can increase home visits to hypertension patients to improve their nutrition knowledge by community health workers.

Salt-restriction-spoons program was effective in the previous trial in China [21], so prevailing free salt-restriction-spoons campaign in the whole country, at the same time strengthening the communitybased education on how to correctly use the spoons might contribute to the decreased salt consumption. Moreover, only supply low salt food in hospitals and schools, a meta-analysis of controlled trials showed that reducing salt intake in children was positively related to the decreased blood pressure and CVD when they grew up. Hospital based education can include patients' family members and friends to increase the effectiveness, which was proved to be effective [36]. It not only helps patients' family members to take care of patients' diets but also makes them realize the importance of healthy eating habits by showing the result of unhealthy diet. And school-based education should better to include students' parents [37], as parents mostly make the food choices for their kids.

\section{Institutional}

Encourage salt industry change to produce low sodium contain salt and seasonings. Food industry and restaurants should change to use low sodium contain salt in food and reduce the use of soy sauce; Supermarkets should be encouraged to sell low sodium contain process food and indicate salt contain food in the package. Design no or low salt food meal sets in restaurants for children (such as KFC and McDonalds). However, private manufacturers are profits seekers; it's hard for them to make the changes voluntarily, so the institutional level changes need government policy support or incentives.

\section{Interpersonal}

Peer support for increasing nutrition knowledge and healthy food choices is useful in reducing salt intake program [38]. Such as familybased education on healthy diet and how to control salt consumption [39]. A study done in African American adolescents in the relation between salt consumption and social support showed that higher levels of emotional support from a family member were positively related to less sodium intake in short-term [40]. Another study was done in the social support and dietary habits among college students also reached the similar conclusion [41].

Parents guidance when their kids are watching TV or using website; encourage to parents to cook at home and less eating crisps and snacks, as parents eating habits will greatly affect their kids $[26,42]$. Establishment and promotion of salt intake assessment methods used for family members which are recommended to Chinese population [28].

\section{Intrapersonal}

Increase personal nutrition knowledge and cooking skills and personal knowledge about the relationship between eating style and NCDs, which is proved to be effective [43]. Correct misconceptions on 
salt intake in general populations and increase patients' self-confidence to control hypertension through reducing salt intake. Use salt restriction spoons when cooking at home to control the salt consumption, as $80 \%$ of salt intake comes from home cooking, so use low sodium salt [7], reduce the usage of soy sauce and salt-containing seasoning during cooking will be effective.

Above all, China is a large country with the biggest population in the world, and different people living in different areas have diverse eating habits and cooking styles. So observational studies are needed to be done before policy makers to make context-specific policies and guidelines in salt intake control. Moreover, salt intake reduction would be more effective with the combined efforts of multi levels rather than individually, as individual eating habits are affected by the environment and the food choices they can have.

\section{Acknowledgement}

This study was financially supported by the Medical Scientific Research Foundation of Guangdong Province, China (WSTJJ20131222440301198203063820).

\section{References}

1. Li Q, Mieke R, Kraus C, Alho J (2009) Ageing of a giant: a stochastic population forecast for China, 2006-2060. Journal of Population Research 26: 21-50.

2. Mozaffarian D, Hao T, Rimm EB, Willett WC, Hu FB (2011) Changes in die and lifestyle and long-term weight gain in women and men. N Engl J Med 364: 2392-2404.

3. Popkin BM, Horton S, Kim S, Mahal A, Shuigao J (2001) Trends in diet nutritional status and diet-related noncommunicable diseases in China and India: The economic costs of the nutrition transition. Nutrition reviews 59: 379 390

4. Amuna P, Zotor FB (2008) Epidemiological and nutrition transition in developing countries: impact on human health and development. Proceedings of the Nutrition Society 67: 82-90.

5. Organization WH (2013) Global action plan for the prevention and control of non-communicable diseases 2013-2020.

6. Ezzati M, Vander Hoorn S, Lawes CM, Leach R, James WP, et al. (2005) Rethinking the "diseases of affluence" paradigm: Global patterns of nutritional risks in relation to economic development. PLoS Med 2: e133.

7. Gu D, Reynolds K, Wu X, Chen J, Duan X, et al. (2002) Prevalence, awareness, treatment and control of hypertension in China. Hypertension 40: 920-927.

8. Gao Y, Chen G, Tian H, Lin L, Lu J, et al. (2013) Prevalence of hypertension in China: A cross-sectional study. PLoS One 8: e65938.

9. Forte J, Miquel JM, Miquel MJ, de Paudua F, Rose G (1989) Salt and blood pressure: A community trial. Journal of human hypertension 3: 179-184.

10. Wang G, Labarthe D (2011) The cost-effectiveness of interventions designed to reduce sodium intake. J Hypertens 29: 1693-1699.

11. Asaria P, Chisholm D, Mathers C, Ezzati M, Beaglehole R (2007) Chronic disease prevention: health effects and financial costs of strategies to reduce salt intake and control tobacco use. Lancet 370: 2044-2053.

12. Ma GS (2008) The salt consumption of residents in China. Chinese Journal of Prevention and Control of Chronic Non-Communicable Diseases 16: 331-333.

13. Du S, Batis C, Wang H, Zhang B, Zhang J, et al. (2014) Understanding the patterns and trends of sodium intake, potassium intake and sodium to potassium ratio and their effect on hypertension in China. The American journal of clinical nutrition 99: 334-343.

14. McLeroy KR, Bibeau D, Steckler A, Glanz K (1988) An ecological perspective on health promotion programs. Health Educ Q 15: 351-377.

15. Ritz E (2006) Salt--friend or foe? Nephrol Dial Transplant 21: 2052-2056

16. Li Y, Zhang J, Shi XM, Linag XF (2013) [A cross-sectional survey on the efficacy of 'China Healthy Lifestyle for All' in 2012]. Zhonghua liu xing bing xue za zh 34: 869-873.

17. Li N, Yan LL, Niu W, Labarthe D, Feng X (2013)A large-scale cluster randomized trial to determine the effects of community-based dietary sodium reduction- the China Rural Health Initiative Sodium Reduction Study. American heart journal 166: 815-822

18. Daniels C, JNeedham, NK Menzies (1996) Science and civilisation in China: Volume 6, biology and biological technology, part 3, agro-industries and forestry. Cambridge University Press.

19. Chen PK, TC Chen, RY Tseng (1983) Everything you want to know about Chinese cooking. Barron's.

20. Saelens BE, Sallis JF, Jennifer BB, Diana Chen (2003) Neighbourhood-based differences in physical activity: An environment scale evaluation. American journal of public health 93: 1552-1558.

21. Chen J, Tian Y, Liao Y, Yang S, Li Z, et al. (2013) Salt-restriction-spoon improved the salt intake among residents in China. PLoS One 8: e78963.

22. Wiecha JL, Peterson KE, Ludwig DS, Kim J, Sobol A, et al. (2006) When children eat what they watch: Impact of television viewing on dietary intake in youth. Archives of Pediatrics \& Adolescent Medicine 160: 436-442.

23. Popkin BM, K Duffey, P Gordon-Larsen (2005) Environmental influences on food choice, physical activity and energy balance. Physiology \& Behavior 86 : 603-613.

24. Chen J, Liao Y, Li Z, Tian Y, Yang S, et al. (2013) Determinants of salt-restrictionspoon using behavior in China: Application of the health belief model. PLoS One 8: e83262.

25. Feunekes GI, de Graaf, Meyboom S, Van Staveren WA (1998) Food choice and fat intake of adolescents and adults: Associations of intakes within social networks. Preventive medicine 27: 645-656.

26. Gibson E, J Wardle, C Watts (1998) Fruit and vegetable consumption, nutritional knowledge and beliefs in mothers and children. Appetite 31: 205-228.

27. Reinaerts E, de Noojier J, Candle M, de Vries N (2007) Explaining schoo children's fruit and vegetable consumption: the contributions of availability, accessibility, exposure, parental consumption and habit in addition to psychosocial factors. Appetite 48: 248-258.

28. Zhao F, Zhang P, Zhang L, Niu W, et al. (2015) Consumption and sources of dietary salt in family members in Beijing. Nutrients 7: 2719-2730.

29. Campbell N, Neal B, MacGregor G (2011) Interested in developing a national programme to reduce dietary salt and quest. Journal of human hypertension 25: 705-710.

30. Smith-Spangler CM, Juusola JL, Enns EA, Owens DK, Garber AM (2010) Population strategies to decrease sodium intake and the burden of cardiovascular disease: A cost-effectiveness analysis. Annals of Internal Medicine 152: 481-487.

31. Kawasaki T, Delea CS, Bartter FC, Smith H (1978) The effect of high-sodium and low-sodium intakes on blood pressure and other related variables in human subjects with idiopathic hypertension. The American Journal of Medicine 64 193-198.

32. Geleijnse JM, Witteman JC, Bak AA, den Breeijen JH, Grobbee DE (1994) Reduction in blood pressure with a low sodium, high potassium, high magnesium salt in older subjects with mild to moderate hypertension. BMJ 309 : 436-440.

33. Sacks FM, Svetkey LP, Vollmer WM, Appel LJ, Bray GA, et al. (2001) Effects on blood pressure of reduced dietary sodium and the dietary approaches to stop hypertension (DASH) diet. DASH-Sodium Collaborative Research Group. N Engl J Med 344: 3-10

34. China Salt Substitute Study Collaborative Group (2007) Salt substitution: A lowcost strategy for blood pressure control among rural Chinese. A randomized, controlled trial. J Hypertens 25: 2011-2018.

35. Cappuccio FP, Capewell S, Lincoln P, McPherson K (2011) Policy options to reduce population salt intake. BMJ 343: d4995

36. Paul S (2008) Hospital discharge education for patients with heart failure: What really works and what is the evidence? Crit Care Nurse 28: 66-82.

37. Perez-Rodrigo C, Aranceta J (2001) School-based nutrition education: Lessons learned and new perspectives. Public Health Nutrition 4: 131-140.

38. Wilcox S, Castro C, King AC, Housemann R, Brownson RC (2000) Determinants of leisure time physical activity in rural compared with urban older and ethnically diverse women in the United States. J Epidemiol Community Health 54: 667672 
Citation: Li M, Zhang L, Shi J (2016) To Live Long, Eat Less Salt: Salt Intake Reduction Promotion and Hypertension Control in China. Health Care: Current Reviews 4: 169. doi: 10.4172/2375-4273.1000169

Page 5 of 5

39. Mu J, Lui Z, Liu F, Xu X, Liang Y, et al. (2009) Family-based randomized trial to detect effects on blood pressure of a salt substitute containing potassium and calcium in hypertensive adolescents. American Journal of Hypertension 22: 943-947.

40. Wilson DK, G Ampey-Thornhill (2001) The role of gender and family support on dietary compliance in an African American adolescent hypertension prevention study. Annals of Behavioral Medicine 23: 59-67.
41. Gruber KJ (2008) Social support for exercise and dietary habits among college students. Adolescence 43: 557-575.

42. Taras HL, Sallis JF, Patterson TL, Nader PR, Nelson JA (1989) Television's influence on children's diet and physical activity. J Dev Behav Pediatr 10: 176-180.

43. Fitzgerald N, K Spaccarotella (2009) Barriers to a healthy lifestyle: from individuals to public policy-an ecological perspective. Journal of extension 47: 1-8. 